\title{
Videoconferencing and Multiple Sclerosis Management: Stopgap or Stay Tuned?
}

\author{
Paul S. Giacomini, Jiwon Oh, Sarah A. Morrow, Philippe Beauchemin, \\ Fraser Clift, Virginia Devonshire
}

Keywords: Multiple sclerosis, Clinical practice, Videoconferencing, Video consult

doi:10.1017/cjn.2021.113

Can J Neurol Sci. 2022; 49: 402-405

\section{INTRODUCTION}

Videoconferencing has emerged as an important telehealth tool to ensure ongoing evaluations of patients during the current COVID-19 pandemic. Indeed, the Canadian Medical Association, the Royal College of Physicians and Surgeons, and the College of Family Physicians of Canada have recently issued a Virtual Care Playbook for clinicians as a guide to incorporating videoconferencing into clinical practice, outlining the technology requirements, and providing sample disclosure and consent forms. ${ }^{1}$

The use of videoconferencing in the specialist management of chronic neurological diseases was not specially addressed in the Virtual Care Playbook. Accordingly, a group of six Canadian neurologists convened a virtual meeting to discuss the role of videoconferencing in the management of patients with multiple sclerosis. The group represented multiple sclerosis (MS) clinicians from across Canada and comprised the directors of the three largest Canadian MS clinics (JO, PSG, VD) and the founder of the first MS cognitive clinic in Canada (SM). As MS clinics have perforce adopted videoconferencing during the pandemic, the objective was to discuss some of its strengths and limitations as a necessary first step to determining the role of this technology in MS clinical practice in the years ahead.

\section{DiAgNOSIS}

MS is a clinical diagnosis that requires thorough historytaking and a neurological examination by a clinician experienced in MS. ${ }^{2}$ The standard of care is that all new diagnoses of a demyelinating syndrome are made during an in-person visit with a neurologist. A thorough neurological examination is needed to rule out the many MS mimics to arrive at an accurate diagnosis. In some clinical situations, such as radiologically isolated syndrome or clinically isolated syndrome, a short delay until an in-person visit and MRI can be arranged may be acceptable. If the patient lives in a remote community, a local clinician may manage the patient with consultation from a neurologist; a 'video triage' may ascertain if an in-person specialist follow-up is required. An in-person visit should be prioritized in the event of aggressive or highly active relapsing MS (RMS), which may result in rapid deterioration and for which the window of opportunity for effective treatment is narrow.

\section{Treatment Decisions}

It is the opinion of this group that a disease-modifying therapy (DMT) can be initiated during a videoconference provided the clinician has an established relationship with the patient and the required baseline tests and procedures have been completed. Patients can be adequately counselled remotely about treatments, side-effect management, and safety monitoring requirements. A switch from one first-line agent (i.e. interferon- $\beta$, glatiramer acetate, teriflunomide, dimethyl fumarate) to another is also feasible, especially if a change is being made to accommodate patient preferences.

Escalating therapy due to an inadequate treatment response, worsening disease, or onset of progressive MS generally warrants an in-person consult to document objective confirmation of clinical changes and to establish a baseline examination. Ideally, the in-person visit would combine an assessment of disease changes and a discussion of the need to escalate therapy. The visit would include a detailed review of the different treatment options along with the risks and benefits associated with individual agents. It is the authors' view that an in-person visit enables the clinician to assess the patient's understanding of the treatment options and to better gauge their emotional response to the news that their condition is deteriorating.

Videoconferencing can be extremely useful for routine follow-ups to accomplish a focussed set of tasks, such as checking on the patient's overall health status and quality of life; providing instruction on a medication (e.g. dose titration); monitoring adherence to the treatment regimen; and evaluating symptoms. It may be possible to safely initiate symptomatic medications for urinary symptoms, spasticity, and fatigue as long as a discussion about the goals of therapy and potential side effects can be adequately performed remotely. Prescribing

From the Montreal Neurological Institute and Hospital, Montreal, Québec, Canada (PSG); St. Michael's Hospital, Toronto, Ontario, Canada (JO); London Health Sciences Centre, London, Ontario, Canada (SAM); CHU de Québec, Québec City, Québec, Canada (PB); Memorial University, St. John's, Newfoundland-Labrador, Canada (FC); and University of British Columbia, Vancouver, British Columbia, Canada (VD)

Received January 20, 2021. Final Revisions Submitted April 22, 2021. Date of Acceptance May 10, 2021.

Correspondence to: Paul Giacomini, MD, $\operatorname{FRCP}(C)$, Director, Multiple Sclerosis Clinic, Montreal Neurological Institute, 3801 University, room 111, Montreal, Quebec, Canada, H3A 2B4. Email: paul.giacomini@mcgill.ca 
medications with abuse potential, such as opioids or cannabis, is not recommended during a video conference call, in accordance with recommendations from provincial Colleges of Physicians and Surgeons.

\section{Patient Evaluation}

When using videoconferencing to obtain an update on the patient's well-being and current functional status, a structured interview is recommended to assess patients' self-reported MS symptoms (e.g. fatigue, pain, bowel/bladder symptoms). It is useful for patients to complete a symptom checklist (e.g. Multiple Sclerosis Impact Scale) prior to the consult (see Links below). Careful questioning can provide some indication about changes in day-to-day functioning, such as difficulty walking a distance that was previously attainable, that will give some indication that ambulation, muscle strength, or endurance may have worsened. An in-patient visit is advised if screening reveals evidence of a relapse or symptom worsening, most notably if the pattern of reported symptoms is inconsistent with previous findings. Supplemental information to assess disability remotely may be obtained from patient-reported outcome measures, such as the Patient-Determined Disease Steps (PDDS) (see Links), which is correlated with the Expanded Disability Status Scale (EDDS); or wearable devices (e.g. Fitbit) that provide continuous monitoring of steps per day. Step counting has been shown to be moderately correlated with other walking measures, but the substantial variability in results (e.g. EDSS $6.0=$ range 1097-7152 steps) limits its clinical usefulness. ${ }^{3}$

Videoconferencing may provide useful information about the patient's neurocognitive status beyond what can be obtained by telephone. In the authors' experience, patients can be screened for symptoms of stress, depression and anxiety, or difficulties coping. An in-person visit may be warranted if there are significant problems.

An adequate assessment of cognitive function may be more challenging. Evaluations may be confounded by interruptions in audio or video transmission and distractions in the patient's home environment. Objective testing (e.g. Symbol Digit Modality Test; CogEval Processing Speed Test) may be employed but is most useful to evaluate cognitive changes longitudinally provided a baseline has been previously obtained. Patient self-reporting of cognitive symptoms may be unreliable; it may be more useful to inquire about changes related to employment (e.g. comprehension problems during a meeting, difficulty with deadlines, recent job loss) or daily activities (e.g. difficulty planning a meal or multitasking household tasks) that may indicate overt cognitive impairment.

The Virtual Care Playbook states that most neurological symptoms are not amenable to virtual care, ${ }^{1}$ and it is the authors' view that videoconferencing is generally not adequate for evaluating neurological signs in MS patients. A virtual neurological exam may be feasible for some conditions (e.g. movement disorders, stroke) ${ }^{4}$ and may be appropriate for patients who do not require a detailed neurological examination (e.g. epilepsy, chronic headache). Remote examination has been shown to be poorer than in-person examination for assessments involving brain stem, cerebellar, and sensory functional systems in MS patients. ${ }^{5}$ Visual acuity and direct visualization of the optic nerve, eye movements (e.g. nystagmus), reflexes, gait, muscle tone and strength, and asymmetry of strength and sensation are difficult to evaluate properly during a virtual assessment. Gait assessments are generally not feasible unless the patient has help positioning the webcam; there is a risk of falls in patients with impairments in ambulation, balance, or coordination. A further consideration is that third-party payors often require an in-person assessment for insurance renewals.

\section{Patient Selection}

As stated in the Virtual Care Playbook, videoconferencing must not compromise the standard of care. ${ }^{1}$ In MS management, videoconferencing appears to be most appropriate for routine follow-up appointments for MS patients with stable disease, a view that is supported by a recent survey of outpatient neurologists. ${ }^{6}$ For the MS population, videoconferencing can address current health status (relapses, symptoms), provide information and reassurance, manage minor problems or concerns that have emerged (e.g. treatment-related adverse effects), and monitor treatment adherence.

The current standard of care for patients who experience relapses or clinical worsening is an in-person visit. Relapse assessment generally requires a hands-on neurological examination and in-person testing by a skilled clinician to ascertain the degree of disability and recovery. Such assessments will determine the need for acute treatment (e.g. corticosteroids); if an MRI would be informative; whether a treatment escalation is required; and if the patient would benefit from additional medications or interventions to manage worsening symptoms.

Videoconferencing is more likely to be satisfactory if the physician-patient relationship is already well established. As MS is a lifelong illness, regular in-person visits from the outset are essential to establish a therapeutic rapport early on. Periodic adjustments to the shared treatment plan may then be implemented in person or via videoconferencing in accordance with changing clinical circumstances.

\section{Requirements for a Video Consult}

The technical requirements for videoconferencing have been well described in the Virtual Care Playbook. Accessibility devices may be required for MS patients with manual dexterity problems or cognitive difficulties. It should be noted that some patients may lack the financial means to obtain equipment or have limited access to a high-speed internet connection. Indeed, a majority of households in rural and indigenous communities in Canada lack access to the bandwidth (50/10 Mbps) required to support videoconferencing (https://crtc.gc.ca/eng/internet/ internet.htm).

\section{The Future of Videoconferencing in MS Management}

Videoconferencing has significant potential to improve healthcare access and continuity of care, but there are at least three important limitations that will need to be addressed before the technology can be adopted more widely in the post-pandemic management of MS.

Telehealth initiatives originated with the need to improve access to healthcare for patients living in remote communities. This is particularly important in MS care since many patients do not have access to a local neurologist. However, there is a need 
for videoconferencing platforms to improve accessibility for people with physical and cognitive impairments. In addition, there is an urgent need for high-speed internet service to be made available to rural and remote communities. Without these changes, the technologies intended to improve access will continue to impose barriers of their own for those most requiring care.

Second, provincial health plans currently reimburse videoconferences on par with in-person visits, which may change once the pandemic has ended. Provincial governments and medical associations will need to identify where videoconferencing is most effectively used based on the available evidence, define medicolegal aspects, provide standards on the technologies that can be employed, and negotiate a fee structure that will adequately compensate healthcare practitioners providing services remotely.

Third, there is the need to streamline the administrative processes involved in delivering videoconferencing, such as linking forms and requisitions to electronic medical records, automating electronic consents, forwarding prescriptions to pharmacies, and coordinating patient follow-ups with nurses and other healthcare providers. Some ad hoc measures have been developed in response to the pandemic but a more standardized, systematic approach is required. Increased institutional support will also be required to provide equipment, resources, and clerical services to clinicians who want to reach their patients by videoconferencing.

Videoconferencing has been an important stopgap measure for maintaining communications between neurologists and their MS patients during the COVID-19 pandemic. This has provided an opportunity for clinicians to rethink how healthcare may be more optimally delivered in the post-pandemic setting and more research will be needed to identify how videoconferencing may be usefully employed. At present, videoconferencing can be technically challenging for some MS patients and in many instances, a telephone call, although less informative, can be more easily arranged.

Videoconferencing will continue to be an important tool postpandemic. For stable MS patients, in-person visits could alternate with a videoconference so that the person can be seen every six months; this alternating schedule would not increase the overall number of visits, could reduce overall healthcare costs, and might be more convenient for patients, especially those with significant disability or who live at a distance from their providers. Videoconferencing would be especially useful for addressing more routine issues and concerns, which could then allow for more in-depth assessment at the annual clinic visit. Such an approach to videoconferencing, at the clinician's discretion and with the patient's consent, could expand access to healthcare services while reducing some of the costs and inconveniences of clinic visits that unnecessarily add to the burden of care for patients living with MS.

\section{LiNKS}

- My MS Companion patient questionnaire: www.msinthe21 stcentury.com/en/resources.

- Patient-Determined Disease Steps (PDDS) scale: www. nationalmssociety.org/NationalMSSociety/media/MSNational Files/Brochures/10-2-3-28-Disease_Steps_Form.pdf.
- Multiple Sclerosis Impact Scale: www.mstrust.org.uk/sites/ default/files/MSIS-29.pdf.

- CogEval Processing Speed Test : https://multiplesclero sisacademy.org/wp-content/uploads/sites/3/2018/11/P-MSFCogEval-Handout-2018-GB-IE-Biogen-02528.pdf. Requires download to an iOS device.

- American Medical Association digital health playbook: www.ama-assn.org/amaone/ama-digital-health-implementationplaybook.

\section{ACKNOWLEDGEMENTS}

The authors would like to thank Anne Morinville, $\mathrm{PhD}$, for her support and encouragement and acknowledge the assistance of Steven Manners of Communications Lansdowne, whose help was made possible through funding from Novartis Pharmaceuticals Canada Inc.

\section{Disclosures}

Paul S. Giacomini has received compensation for speaking, advisory board participation or clinical trials from Alexion, Biogen, Bristol Myers Squibb/Celgene, EMD Serono, Novartis, Roche Genentech, Sanofi Genzyme, and Teva.

Jiwon Oh reports research grant funding from MS Society of Canada, Barford, and Love MS Fund of St. Michael's Hospital Foundation, National MS Society, Brain Canada, Biogen Idec, Roche, and EMD Serono; and personal fees for consulting or speaking from Biogen Idec, EMD Serono, Roche, SanofiGenzyme, Novartis, Alexion, and Celgene.

Sarah A. Morrow has received research grants from Biogen Idec, Novartis, and Hoffmann-La Roche; has been an investigator for clinical trials for Genzyme, Novartis, Hoffmann-La Roche, and AbbVie and has received honoraria from Biogen Idec, EMD Serono, Genzyme, Novartis, and Hoffmann-La Roche.

Philippe Beauchemin has participated in advisory boards for Biogen, Alexion, Hoffmann-La Roche, EMD Serono, and Sanofi Genzyme and has received speaker fees from Alexion, Hoffmann-La Roche, Novartis, and Pendopharm.

Fraser Clift has received honoraria from Alexion, Biogen, Bristol Myers Squibb, EMD Serono, Novartis, Hoffmann-La Roche, and Sanofi.

Virginia Devonshire has nothing to disclose.

\section{Statement of Authorship}

All of the authors contributed to the discussions, reviewed all drafts, and approved the final version of this manuscript. Authors did not receive any remuneration for their meeting attendance or review of the manuscript.

\section{REFERENCES}

1. Dermer M. Virtual Care Playbook, Canadian Medical Association, the College of Family Physicians of Canada and the Royal College of Physicians and Surgeons of Canada, March 2020. www.cma.ca/sites/default/files/pdf/Virtual-CarePlaybook_mar2020_E.pdf, accessed 8 April 2021.

2. Thompson AJ, Banwell BL, Barkhof F, et al. Diagnosis of multiple sclerosis: 2017 revisions of the McDonald criteria. Lancet Neurol 2018;17:162-173. 
3. Block VJ, Lizée A, Crabtree-Hartman E, et al. Continuous daily assessment of multiple sclerosis disability using remote step count monitoring. J Neurol 2017;264:316-326.

4. Hussona MA, Mahera M, Chan D, et al. The virtual neurologic exam: instructional videos and guidance for the COVID-19 era. Can J Neurol Sci 2020;47:598-603.
5. Yeroushalmi S, Maloni H, Costello K, Wallin MT. Telemedicine and multiple sclerosis: a comprehensive literature review. J Telemed Telecare 2020;26:400-413.

6. Kristoffersen ES, Sandset EC, Winsvold BS, Faiz KW, Storstein AM. Experiences of telemedicine in neurological out-patient clinics during the COVID-19 pandemic. Ann Clin Transl Neurol 2021;8:440-447. 